\title{
The development of allergic inflammation in a murine house dust mite asthma model is suppressed by synbiotic mixtures of non-digestible oligosaccharides and Bifidobacterium breve M-16V
}

\author{
K. A. T. Verheijden ${ }^{1}$ L. E. M. Willemsen ${ }^{1}$ S. Braber ${ }^{2}$ T. Leusink-Muis ${ }^{1}$ \\ P. V. Jeurink ${ }^{3}$ J. Garssen ${ }^{1,3}$ • A. D. Kraneveld ${ }^{1}$ - G. Folkerts ${ }^{1}$
}

Received: 31 January 2015 / Accepted: 9 May 2015 / Published online: 24 May 2015

(C) The Author(s) 2015. This article is published with open access at Springerlink.com

\begin{abstract}
Purpose The incidence and severity of allergic asthma is rising, and novel strategies to prevent or treat this disease are needed. This study investigated the effects of different mixtures of non-digestible oligosaccharides combined with Bifidobacterium breve M-16V (BB) on the development of allergic airway inflammation in an animal model for house dust mite (HDM)-induced allergic asthma.

Methods BALB/c mice were sensitized intranasally (i.n.) with HDM and subsequently challenged (i.n.) with PBS or HDM while being fed diets containing different oligosaccharide mixtures in combination with $B B$ or an isocaloric identical control diet. Bronchoalveolar lavage fluid (BALF) inflammatory cell influx, chemokine and cytokine concentrations in lung homogenates and supernatants of ex vivo HDM-restimulated lung cells were analyzed.

Results The HDM-induced influx of eosinophils and lymphocytes was reduced by the diet containing the shortchain and long-chain fructo-oligosaccharides and $B B$ $(\mathrm{FF} B B)$. In addition to the HDM-induced cell influx, concentrations of IL-33, CCL17, CCL22, IL-6, IL-13 and IL-5 were increased in supernatants of lung homogenates or BALF and IL- 4 , IFN- $\gamma$ and IL-10 were increased in restimulated lung cell suspensions of HDM-allergic mice. The
\end{abstract}

K. A. T. Verheijden

k.a.t.verheijden@uu.nl

1 Division of Pharmacology, Department of Pharmaceutical Sciences, Faculty of Science, Utrecht University, Universiteitsweg 99, 3584 CG, Utrecht, The Netherlands

2 Division of Veterinary Pharmacy, Pharmacology and Toxicology, Faculty of Veterinary Sciences, Utrecht University, Utrecht, The Netherlands

3 Nutricia Research, Immunology, Utrecht, The Netherlands diet containing FFBB reduced IL-6, IFN- $\gamma$, IL-4 and IL-10 concentrations, whereas the combination of galacto-oligosaccharides and long-chain fructo-oligosaccharides with $B B$ was less potent in this model.

Conclusion These findings show that synbiotic dietary supplementation can affect respiratory allergic inflammation induced by HDM. The combination of FFBB was most effective in the prevention of HDM-induced airway inflammation in mice.

Keywords House dust mite · Asthma - Allergy ·

Oligosaccharides $\cdot$ Bifidobacterium breve

\begin{tabular}{|c|c|}
\hline \multicolumn{2}{|c|}{ Abbreviations } \\
\hline AHR & Airway hyperresponsiveness \\
\hline BALF & Bronchoalveolar lavage fluid \\
\hline$B B$ & Bifidobacterium breve M-16V \\
\hline DC & Dendritic cell \\
\hline $\mathrm{FF} B B$ & $\begin{array}{l}\text { Short-chain and long-chain fructo-oligosaccharide } \\
\text { with Bifidobacterium breve } \mathrm{M}-16 \mathrm{~V}\end{array}$ \\
\hline FOS & Fructo-oligosaccharide \\
\hline GFBB & $\begin{array}{l}\text { Galacto-oligosaccharide and long-chain fructo-oli- } \\
\text { gosaccharide with Bifidobacterium breve } \mathrm{M}-16 \mathrm{~V}\end{array}$ \\
\hline GOS & Galacto-oligosaccharide \\
\hline HDM & House dust mite \\
\hline i.n & Intranasally \\
\hline ILC2 & Innate lymphoid cell type 2 \\
\hline $\mathrm{R}_{\mathrm{L}}$ & Lung resistance \\
\hline
\end{tabular}

\section{Introduction}

Allergic asthma is a chronic disease that affects around 235 million people worldwide. Asthma is not only a health problem for developed countries but also for developing 
countries, and the prevalence is still increasing [1]. The disease is characterized by impaired lung function as well as airway inflammation containing high numbers of eosinophils [2]. House dust mite (HDM) is one of the well-known allergens that can trigger allergic diseases such as asthma [3]. The inflammatory response, mainly the recruitment of eosinophils to the airway tissue and the production of cytokines and chemokines, is initiated by HDM-allergenspecific Th2 cells [4]. During sensitization, allergens can directly trigger or stimulate airway epithelial cells, which subsequently release different cytokines (e.g., IL-33) and chemokines (e.g., CCL20) [5]. In response to IL-33, group 2 innate lymphoid cells (ILC2) will proliferate and produce IL-4, -5 and -13 [6]. In addition, IL-33 also induces the maturation of dendritic cells (DC) having Th2-inducing polarizing capacities [7]. These specific DCs release Th2inducing chemokines such as CCL17 and CCL22. The activated DCs take up the HDM allergen and induce the development of naïve T cells into antigen-specific Th2 cells in the local lymph nodes. Subsequently, these Th2 cells migrate back to the lung mucosal tissue. After encountering DC presenting the antigen in the airways, these effector Th2 cells will release IL-4, IL-5 and IL-13 [5], which can trigger allergic symptoms and eosinophilic inflammation [8]. Novel preventive and/or therapeutic approaches are needed to prevent and/or treat asthmatic disorders, while current treatment is still not sufficient and has considerable side effects. Results from animal and human studies suggest that changes in the intestinal microbiota can contribute to the development of asthma. Indeed different animal studies have shown that the gut microbiota affects systemic immune function [9-12]. Since the composition of the microbiota is important for a balanced immune response, adapting the microbiota using oligosaccharides either or not combined with beneficial bacteria may help to protect against the development of allergic disease. For example, treatment with $B$. breve M-16V was found to suppress airway inflammation and treatment with Lactobacillus rhamnosus also reduced lung resistance in a murine ovalbumininduced asthma model [13]. In addition to the direct use of probiotics, also specific non-digestible oligosaccharides such as galacto-oligosaccharides (GOS) and short-chain and long-chain fructo-oligosaccharides (scFOS and lcFOS, respectively) can be administered to support growth and/ or activity of bifidobacteria and lactobacilli $[9,14,15]$. In previous studies, we have shown that GOS alone are capable of suppressing HDM-induced airway hyperresponsiveness, airway eosinophilia and Th2-related cytokine and chemokine concentrations in the lung [16]. Furthermore, the combination of GFBB or FFBB with acidic oligosaccharides reduced allergic responses in food allergic mice and suppressed airway inflammation in a mouse model for ovalbumin-induced asthma, respectively $[17,18]$. In mice affected with food allergy, GFBB was more effective than GF or $B B$ alone [17]. When GFB $B$ was used in patients suffering from HDM-induced allergy and asthma, the peak expiratory flow was increased and the production of systemic Th2 cytokines (IL-4, IL-5 and IL-13) was reduced. However, in this study, no effect on bronchial allergic inflammation was demonstrated [19]. GOS are prepared from lactose derived from cow's milk. Fructo-oligosaccharides, obtained from chicory root, provide a vegetable source of prebiotic oligosaccharides and may be used as an alternative for GOS in the synbiotic mixture. The aim of this study was to investigate the effect of two different synbiotic mixtures, GFBB and FFBB, on pulmonary inflammation, cytokine and chemokine concentrations in lungs and BALF of HDM-induced asthmatic mice.

\section{Materials and methods}

\section{Mice}

Six- to eight-week-old, specific pathogen-free, male BALB/c mice (Charles River, The Netherlands) were housed under bio-contained sterile conditions using HEPA $^{\circledR}$-filtered isocages ${ }^{\circledR}$ (Tecniplast, Italy). Food and water were provided ad libitum. All animal experiments were conducted in compliance with the Guidelines of the Ethical Committee on the Use of Laboratory Animals of the Utrecht University (DEC 2013.II.01.003).

\section{Murine HDM-induced asthma model}

While under isoflurane anesthesia, BALB/c mice were intranasally (i.n.) sensitized with $1 \mu \mathrm{g} \mathrm{HDM} / 40 \mu \mathrm{L}$ PBS (Greer Laboratories, USA) on day 0 and challenged once a day for 5 consecutive days (day 7-11) with PBS (phosphate buffered saline, control, HDM-PBS) or $10 \mu \mathrm{g} \mathrm{HDM} / 40 \mu \mathrm{L}$ PBS (HDM-HDM) [20]. Mice were ad libitum fed the control diet (Research Diet Services, The Netherlands; AIN93G, contr), a diet containing a $1 \% \mathrm{w} / \mathrm{w}$ 9:1 mixture of GOS (Vivinal ${ }^{\circledR}$ GOS syrup with approximately $59 \%$ GOS, $21 \%$ lactose, $19 \%$ glucose and $1 \%$ galactose on dry matter (dry matter of $75 \%$ ); FrieslandCampina Domo, The Netherlands) and long-chain fructo-oligosaccharides (lcFOS, Orafti ${ }^{\circledR}$ HP with approximately $\sim 100 \%$ inulin, $\mathrm{DP} \geq 23$; Beneo, Belgium; GF) or a $1 \% \mathrm{w} / \mathrm{w} 1: 1 \mathrm{mix}-$ ture of short-chain fructo-oligosaccharides $\left(\mathrm{Orafti}^{\circledR} \mathrm{P95}\right.$, with approximately $95 \%$ oligofructose content, DP 2-8; Beneo, Belgium) and long-chain fructo-oligosaccharides (FF). Carbohydrates in Vivinal ${ }^{\circledR}$ GOS were compensated isocalorically in the control diet by means of exchange against cellulose (for GOS), lactose (for lactose) and dextrose (for glucose). In case of the fructo-oligosaccharides, 
a

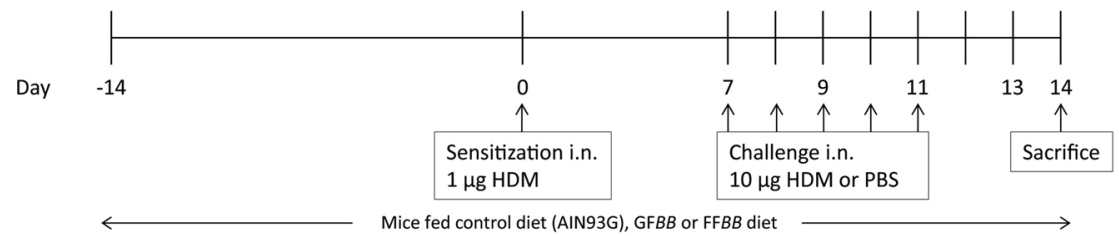

C

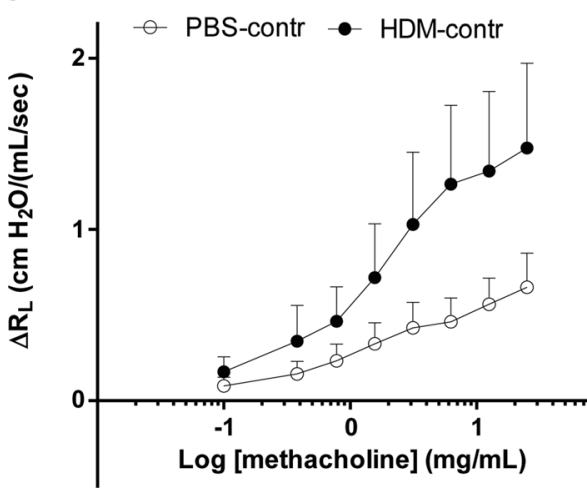

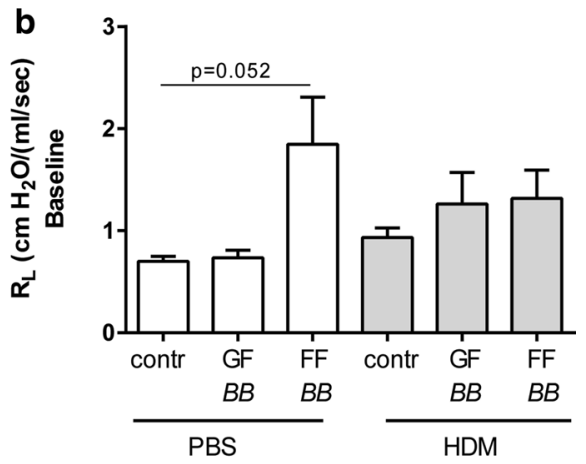

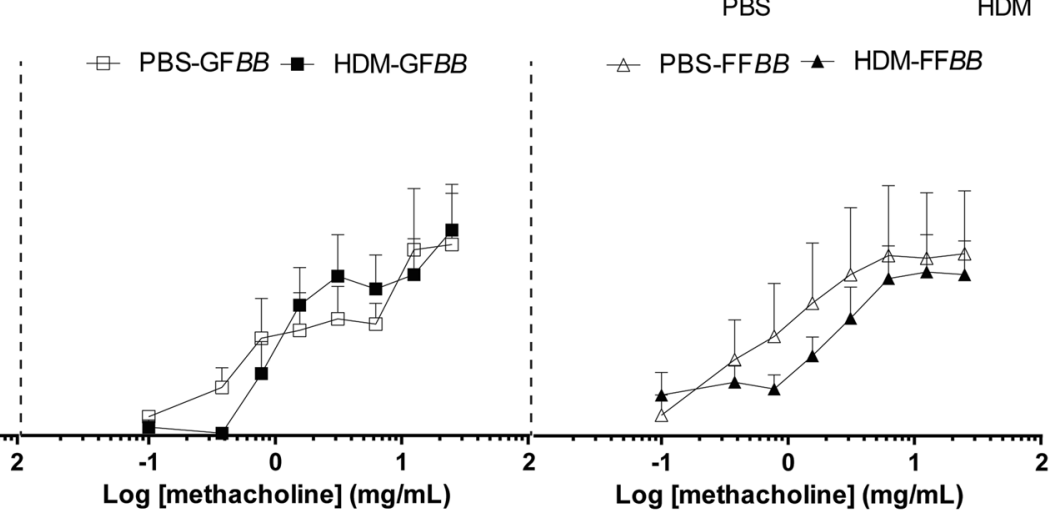

Fig. 1 Treatment protocol of the allergic house dust mite (HDM) asthma model and lung resistance measurement. Male BALB/c mice were sensitized intranasally (i.n.) with HDM on day 0 and were challenged i.n. for five consecutive days with HDM or PBS. Mice were fed the control diet (AIN93G, contr), a diet containing a $1 \%$ w/w 9:1 mixture of GOS and long-chain fructo-oligosaccharide (GF) or $1 \%$ w/w 1:1 mixture of short-chain fructo-oligosaccharide and long-chain fructo-oligosaccharide (FF) both in combination with $2 \times 10 \mathrm{E} 9$ colony-forming units/g Bifidobacterium breve M-16V $(B B)(2 \% \mathrm{w} / \mathrm{w})$. The GFBB and FFBB interventions started 2 weeks prior to sensitiza-

carbohydrates were compensated isocalorically in the control diet by means of exchange against cellulose (for FOS). Both GF and FF diets contained $2 \times 10 \mathrm{E} 9$ colony-forming units/g (2\% w/w) Bifidobacterium breve M-16V (BB) (Morinaga milk Industry, Japan). The GFBB and FFBB diets were given 2 weeks prior to sensitization and were continuously provided throughout the entire experimental period. Mice were killed on day 14 (see treatment schedule in Fig. 1a).

\section{Airway responsiveness measurement}

Mice were anaesthetized with a mixture of ketamine (Vetoquinol S.A., France; $125 \mathrm{mg} / \mathrm{kg}$ ) and medetomidine (Pfizer, The Netherlands; $0.4 \mathrm{mg} / \mathrm{kg}$ ), intraperitoneally (i.p.). EMKA invasive measurement of dynamic resistance (EMKA Technologies, France) in response to increasing doses of methacholine (acetyl- $\beta$-methyl-choline chloride, Sigma-Aldrich, The Netherlands; $0-25 \mathrm{mg} / \mathrm{mL}, 10 \%$ puff tion and continued during the entire experiment. All mice were killed on day 14 (a). Basal airway resistance $\left(\mathrm{R}_{\mathrm{L}}\right)(\mathbf{b})$ and $\Delta \mathrm{R}_{\mathrm{L}}$ after baseline correction in response to increasing doses of methacholine were measured on day 14 (c). HDM-PBS: HDM-sensitized and PBS-challenged mice (white bars), HDM-HDM: HDM-sensitized and HDMchallenged mice (gray bars), Contr: control diet, GFBB: mixture of GOS and long-chain fructo-oligosaccharide with Bifidobacterium breve $\mathrm{M}-16 \mathrm{~V}$ diet, $\mathrm{FF} B B$ : mixture of short-chain fructo-oligosaccharide and long-chain fructo-oligosaccharide with Bifidobacterium breve $\mathrm{M}-16 \mathrm{~V}$ diet

for $10 \mathrm{~s}$.) was used to determine the lung function on day 14 [13]. Basal airway resistance values of each individual mouse as measured prior to methacholine exposure were deducted from the resistance as measured upon methacholine exposure $(\Delta$ R; Fig. $1 \mathrm{~b}-\mathrm{c})$.

\section{Bronchoalveolar lavage}

Mice were killed on day 14 using an intraperitoneal overdose of pentobarbital $\left(600 \mathrm{mg} / \mathrm{kg}\right.$, Nembutal ${ }^{\mathrm{TM}}$, Ceva Santé Animale, The Netherlands). Lungs were lavaged with $1 \mathrm{~mL}$ of pyrogen-free saline $\left(0.9 \% \mathrm{NaCl}, 37{ }^{\circ} \mathrm{C}\right)$ supplemented with protease inhibitor cocktail tablet (Complete Mini, Roche Diagnostics, Germany). The supernatant of the first $\mathrm{mL}$ was used for cytokine and chemokine analyses, followed by three lavages with $1 \mathrm{~mL}$ saline solution $(0.9 \%$ $\left.\mathrm{NaCl}, 37{ }^{\circ} \mathrm{C}\right)$. The BALF cells were centrifuged (400 g, $5 \mathrm{~min}$ ), and pellets of the four lavages were pooled; total numbers of BALF cells were counted using a Bürker-Türk 
chamber (magnification $100 \times$ ). Cytospin were stained with Diff-Quick (Merz \& Dade A.G., Switzerland) for differential BALF cell counts. Numbers of macrophages, lymphocytes, neutrophils and eosinophils were scored using light microscopy [21].

\section{Preparation of lung homogenates}

Lung samples were homogenized in $1 \%$ Triton $\mathrm{X}-100$ (Sigma-Aldrich)/PBS containing protease inhibitor (Complete Mini, Roche Diagnostics, Germany) using a Precellys 24 tissue homogenizer (Bertin Technologies, France). Homogenates were centrifuged at 14,000 rpm for $5 \mathrm{~min}$, and supernatants were collected. The protein concentration of each sample was assayed using the Pierce BCA protein assay kit standardized to BSA according to the manufacturer's protocol (Thermo Fisher Scientific, USA). The homogenates were diluted to a final concentration of $1 \mathrm{mg}$ protein/mL [22, 23].

\section{Ex vivo lung restimulation with house dust mite}

Lung cell suspensions were prepared by cutting the lung into small pieces and by adding digestion buffer, containing DNase I and Collagenase A (Roche Diagnostics, Germany), for $30 \mathrm{~min}$. The digestion was stopped using fetal calf serum (FCS; Hyclone Laboratories, USA). The lung pieces were transferred toward a 70- $\mu \mathrm{m}$ nylon cell strainer (BD Biosciences, The Netherlands) and rinsed with $10 \mathrm{~mL}$ RPMI. Cells were washed and resuspended in RPMI-1640 culture medium (Lonza, USA) supplemented with $10 \%$ heat-inactivated FCS and $0.1 \%$ penicillin-streptomycin solution (Sigma-Aldrich). Total number of cells was calculated using a Beckman Z1 coulter ${ }^{\circledR}$ Particle Counter (Beckman, USA). Lung cells $\left(4 \times 10^{5}\right.$ cells/well) were cultured in a Greiner bio-one CellSTAR 96-well U-bottom plate (Greiner Bio-One B. V., The Netherlands) in medium with or without $50 \mu \mathrm{g} / \mathrm{mL}$ HDM (Greer Laboratories, USA). The supernatant was harvested after 4 days of culture at $37{ }^{\circ} \mathrm{C}$ in $5 \% \mathrm{CO}_{2}$ and stored at $-20{ }^{\circ} \mathrm{C}$ until further analysis [13].

\section{Measurement of cytokines}

A standard Th1/Th2/Th17 assay (BD Biosciences, The Netherlands) was used to determine cytokine concentrations in lung homogenates and supernatants of lung restimulation according to the manufacturer's instructions. IL-33, CCL17, CCL20 and CCL22 were measured with a DuoSet ELISA (R\&D Systems), and IL-13 and IL-5 with a Ready-SET-Go! ${ }^{\circledR}$ ELISA (eBioscience, USA). The concentrations of these cytokines were expressed as $\mathrm{pg} / \mathrm{mg}$ protein in lung homogenates and $\mathrm{pg} / \mathrm{mL}$ in BALF and restimulation supernatants.

\section{Statistical analysis}

Results are presented as mean \pm standard error of mean (SEM). Data were statistically analyzed by one-way ANOVA and post hoc Bonferroni's multiple comparisons test. $P<0.05$ were considered significant. Statistical analyses were conducted using GraphPad Prism software (version 6.04).

\section{Results}

\section{Dietary intervention with FFBB reduced pulmonary eosinophilic inflammation in HDM-allergic mice}

Airway hyperresponsiveness (AHR) to methacholine aerosols was measured at baseline; no differences were observed between the PBS- and HDM-challenged mice fed the different diets (Fig. 1b). In the PBS-challenged mice fed the $\mathrm{FF} B B$ diet, the baseline airway resistance tended to increase compared to PBS mice fed the control diet (Fig. 1b); however, methacholine exposure did not significantly enhance hyperreactivity in these mice as compared to control diet fed mice (Fig. 1c). HDM-challenged mice showed a higher AHR response upon methacholine exposure than PBS-challenged mice, but this did not reach significance (Fig. 1c). HDM challenge in GFBB- or FFBB-fed mice did not increase the AHR response compared to PBSchallenged mice fed the similar diet (Fig. 1c). Furthermore the AHR of HDM-challenged mice fed GFBB or FFBB remained below the AHR of HDM-challenged mice fed the control diet albeit this did not reach significance (Fig. 1c).

BALF was examined to investigate the inflammatory cell influx into the airways of HDM-allergic mice upon dietary intervention with control diet or the synbiotic diets (Fig. 2a). The total number of inflammatory cells in the BALF of HDM-HDM mice fed the control diet was significantly increased when compared to HDM-PBS mice (Fig. 2a). The HDM challenge induced an increase in the number of eosinophils and lymphocytes. The same tendency was shown for macrophages (Fig. 2b-d) compared to the PBS-challenged group. Dietary intervention with FF $B B$ reduced the total number of inflammatory cells in HDM-allergic mice compared to HDM-HDM group fed the control diet (Fig. 2a); this reduction was mainly due to a decrease in the number of eosinophils and lymphocytes (Fig. 2b, c). Dietary intervention with GFBB did not suppress the total number of BALF cells (Fig. 2a) or the differentiated cell numbers (Fig. 2b-d). In this group, the number 
Fig. 2 Dietary intervention with FF $B B$ reduced pulmonary eosinophilic inflammation in the lungs of HDM-allergic mice.

Differentiation of inflammatory cell infiltration in the BALF of house dust mite-allergic mice. HDM-PBS: HDM-sensitized and PBS-challenged mice (white bars), HDM-HDM: HDM-sensitized and HDMchallenged mice (gray bars), Contr: control diet, GFBB: mixture of GOS and long-chain fructo-oligosaccharide with Bifidobacterium breve $\mathrm{M}-16 \mathrm{~V}$ diet, FFBB: mixture of shortchain fructo-oligosaccharide and long-chain fructo-oligosaccharide with Bifidobacterium breve $\mathrm{M}-16 \mathrm{~V}$ diet. Total BALF cells (a), absolute number of eosinophils (b), lymphocytes (c), macrophages (d) and neutrophils (e). Results are shown as mean \pm SEM. Statistical significance of differences by one-way ANOVA and post hoc Bonferroni's multiple comparisons test. $* P<0.05, * * P<0.01$, $* * * P<0.001$ compared to HDM-HDM; ${ }^{\$ \$} P<0.01$, ${ }^{\$ \$} P<0.001,{ }^{\$ \$ \$} P<0.0001$ compared to HDM-PBS group of corresponding diet, $n=6-9$ mice/group
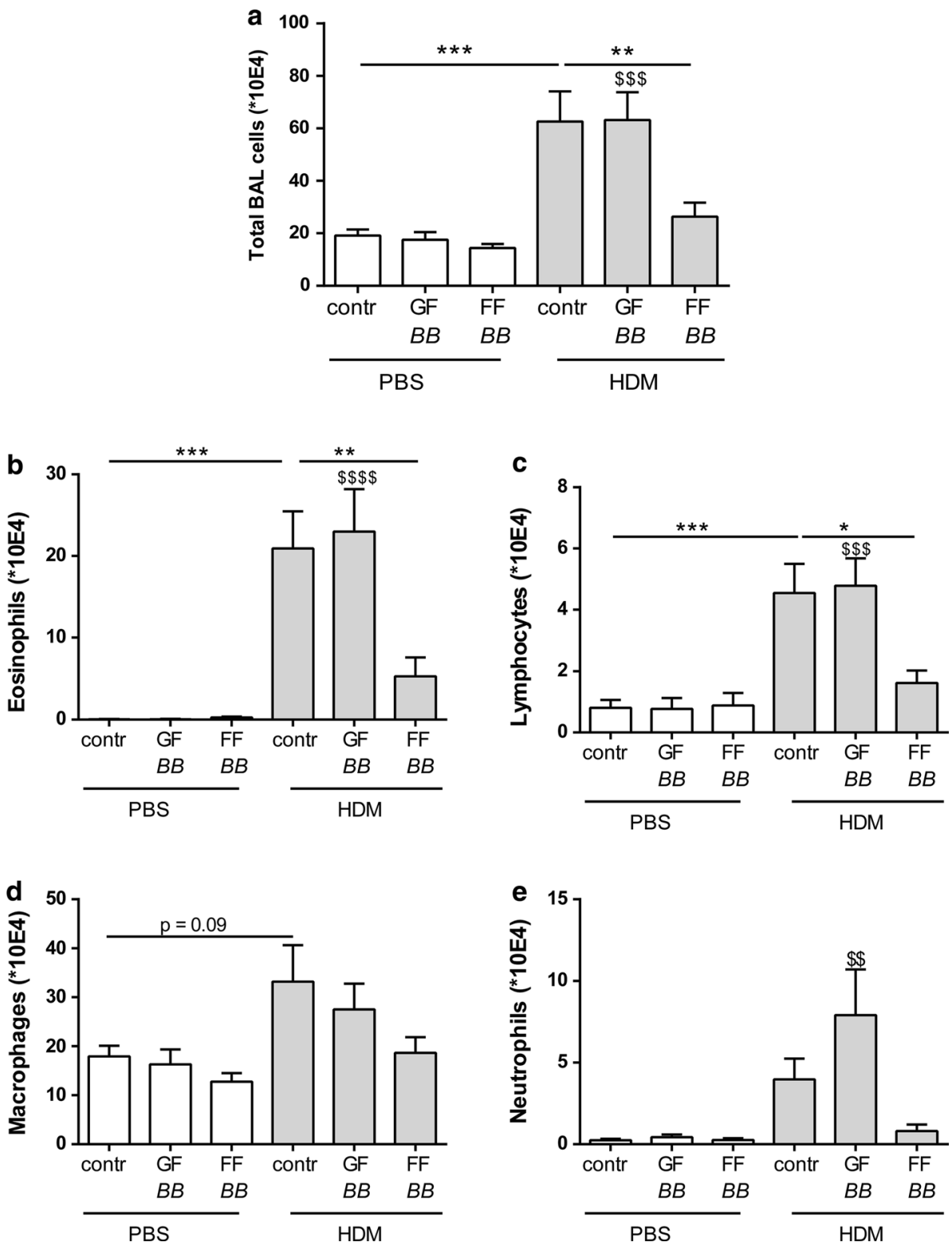

of neutrophils was significantly increased compared to the HDM-PBS control group fed the GFBB diet (Fig. 2e).

\section{Effect of synbiotics on inflammatory and Th2-type cytokines and chemokines in lungs of HDM-allergic mice}

IL-33, CCL20, CCL17 and CCL22 were significantly increased in supernatants of lung homogenates of HDMHDM mice fed the control diet compared to the HDMPBS control group. Dietary interventions with the different synbiotics did not affect these cytokines and chemokines (Fig. 3a-d). IL-6 and IL-13 concentrations in the supernatant of the lung homogenates and IL-5 in BALF of
HDM-HDM mice fed the control diet showed a significant increase compared to the HDM-PBS control group (Fig. 4a, c, d). Dietary intervention with FFBB significantly decreased the HDM allergy-induced increase in IL-6 (Fig. 4a). IL-6 concentrations in lung tissue correlated positively with the total number of BALF cells (Fig. 4b, $r=0.5011, P=0.0288)$. Dietary interventions with the different synbiotics did not affect this HDM-induced increase in pulmonary IL-13 (Fig. 4c), but tended to decrease the concentrations of IL-5 in the BALF (Fig. 4d). IL-5 concentrations in the BALF were positively correlated with the number of lymphocytes (Fig. 4e, $r=0.8257, P=0.0003$ ) and the number of eosinophils in the BALF, respectively (Fig. 4f, $r=0.7292, P=0.0028$ ). 
Fig. 3 IL-33, CCL20, CCL17 and CCL22 in lungs of HDMallergic mice. IL-33 (a), CCL20 (b), CCL17 (c) and CCL22 (d) concentrations were measured in supernatant of lung homogenates. HDM-PBS: HDMsensitized and PBS-challenged mice (white bars), HDM-HDM: HDM-sensitized and HDMchallenged mice (gray bars), Contr: control diet, GFBB: mixture of GOS and long-chain fructo-oligosaccharide with Bifidobacterium breve M-16V diet, FFBB: mixture of shortchain fructo-oligosaccharide and long-chain fructo-oligosaccharide with Bifidobacterium breve M-16V diet. Results are shown as mean \pm SEM. Statistical significance of differences by one-way ANOVA and post hoc Bonferroni's multiple comparisons test. $* P<0.05$,

$* * P<0.01$ compared to HDMHDM $;{ }^{\$} P<0.05,{ }^{\$ \$} P<0.001$ compared to corresponding PBS group of diet, $n=5$ mice/group
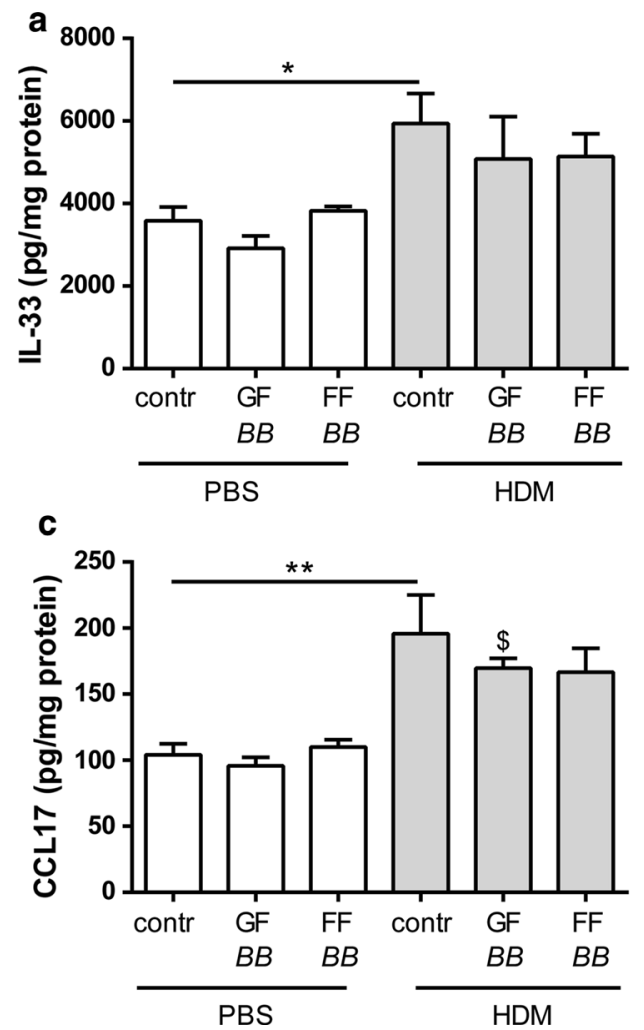

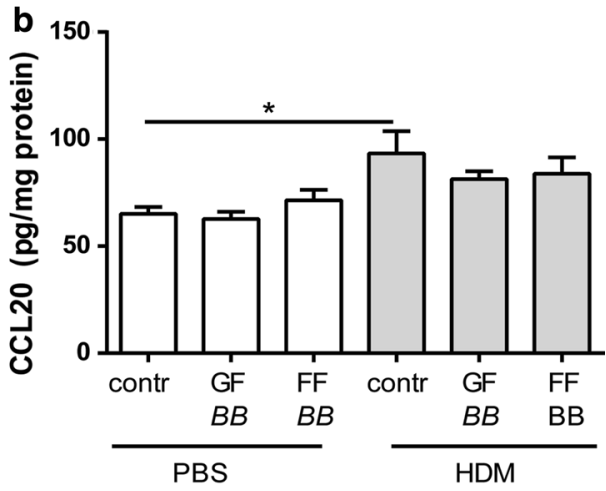

d

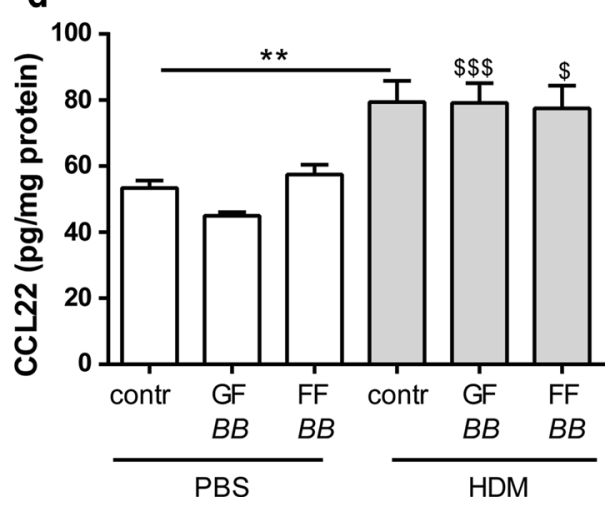

\section{Synbiotic diets suppress $\mathbf{T}$ cell activity in HDM-allergic mice}

To investigate the effects of different synbiotics on allergen-specific cytokine secretion of lung tissue, cell suspensions were ex vivo restimulated with HDM. The concentrations of IL-10, IFN $\gamma$ and IL-4 were significantly increased upon HDM stimulation of lung cells of the HDM-HDM mice fed the control diet, whereas HDM-PBS mice showed no increase compared to medium stimulation (Fig. 5a-c). Dietary intervention with $G F B B$ and FFBB significantly decreased the concentrations of IL-10, while FFBB also decreased IL-4 and IFN $\gamma$ (Fig. 5a-c). Although upon HDM stimulation IL-17A concentrations were increased in the lung cells of HDM-HDM mice fed the control diet, both dietary interventions showed no effect (data not shown).

\section{Discussion}

This is the first study in which anti-inflammatory effects of different oligosaccharide mixtures (e.g., GF or FF) in combination with Bifidobacterium breve M-16V (BB) (synbiotics) were investigated on the development of HDMinduced allergic inflammation in a murine model appropriate to mimic human allergic asthma. To mimic the human features of asthma, murine models for HDM-allergic asthma are commonly used, since HDM is one of the most common allergens associated with human allergic asthma $[24,25]$. In this study, the effects of the synbiotic diets on AHR remain unclear; by contrast, strong protective effects on airway inflammation were observed in HDM-allergic mice. Total inflammatory BALF cell numbers were significantly increased in HDM-HDM-allergic mice when compared to HDM-PBS control mice. In contrast to GFBB, dietary intervention with FFBB significantly decreased the total inflammatory cell number. This decrease was mainly due to the reduction in the number of eosinophils and lymphocytes compared to HDM-HDM mice fed the control diet. Although FFBB was not able to reduce epithelial and DC-related cytokines (IL-33) and chemokines (CCL20, CCL17 and CCL22) known to contribute to allergic sensitization, FFBB decreased the pro-inflammatory cytokine IL-6. IL-6 was found to correlate with the number of total inflammatory cells in the BALF. Furthermore, FFBB reduced Th2-related cytokines (IL-4 and IL-5) and Th1related cytokine IFN- $\gamma$ concentrations in lung homogenates or in supernatants of ex vivo restimulated lung cell suspensions. GFBB was less effective but showed a similar pattern with regard to these parameters, and both $\mathrm{FF} B B$ and the GFBB diet reduced IL-10 concentrations after ex vivo restimulation of lung cells with HDM.

The data show that in particular the FFBB diet was capable of suppressing airway inflammation, whereas it did not suppress Th2-driving mediators such as IL-33, CCL17 and CCL22. Epithelial cells are known to secrete IL-33 upon 
Fig. 4 IL-6, IL-13 and IL-5 in lungs of HDM-allergic mice. IL-6 (a), IL-13 (c) concentrations were measured in supernatant of lung homogenates. IL-5 (d) was measured in the BALF. Correlation of IL-6 in lung homogenates and the total number of BALF cells (b), IL-5 in the BALF and the number of lymphocytes (e) and IL-5 in the BALF and the number of eosinophils (f). HDM-PBS: HDMsensitized and PBS-challenged mice (white bars), HDM-HDM: HDM-sensitized and HDMchallenged mice (gray bars), Contr: control diet, GFBB: mixture of GOS and long-chain fructo-oligosaccharide with Bifdobacterium breve $\mathrm{M}-16 \mathrm{~V}$ diet, FFBB: mixture of short-chain fructo-oligosaccharide and long-chain fructo-oligosaccharide with Bifidobacterium breve M-16V diet. Results are shown as mean \pm SEM. Statistical significance of differences by one-way ANOVA and post hoc Bonferroni's multiple comparisons test. $* P<0.05$, $* * P<0.01$, $* * * * P<0.0001$ compared to HDM-HDM; ${ }^{\$ \$} P<0.01$, $\$ \$ P<0.001$ compared to corresponding PBS group of diet, $n=5-9$ mice/group. Correlation was analyzed using Spearman's correlation test
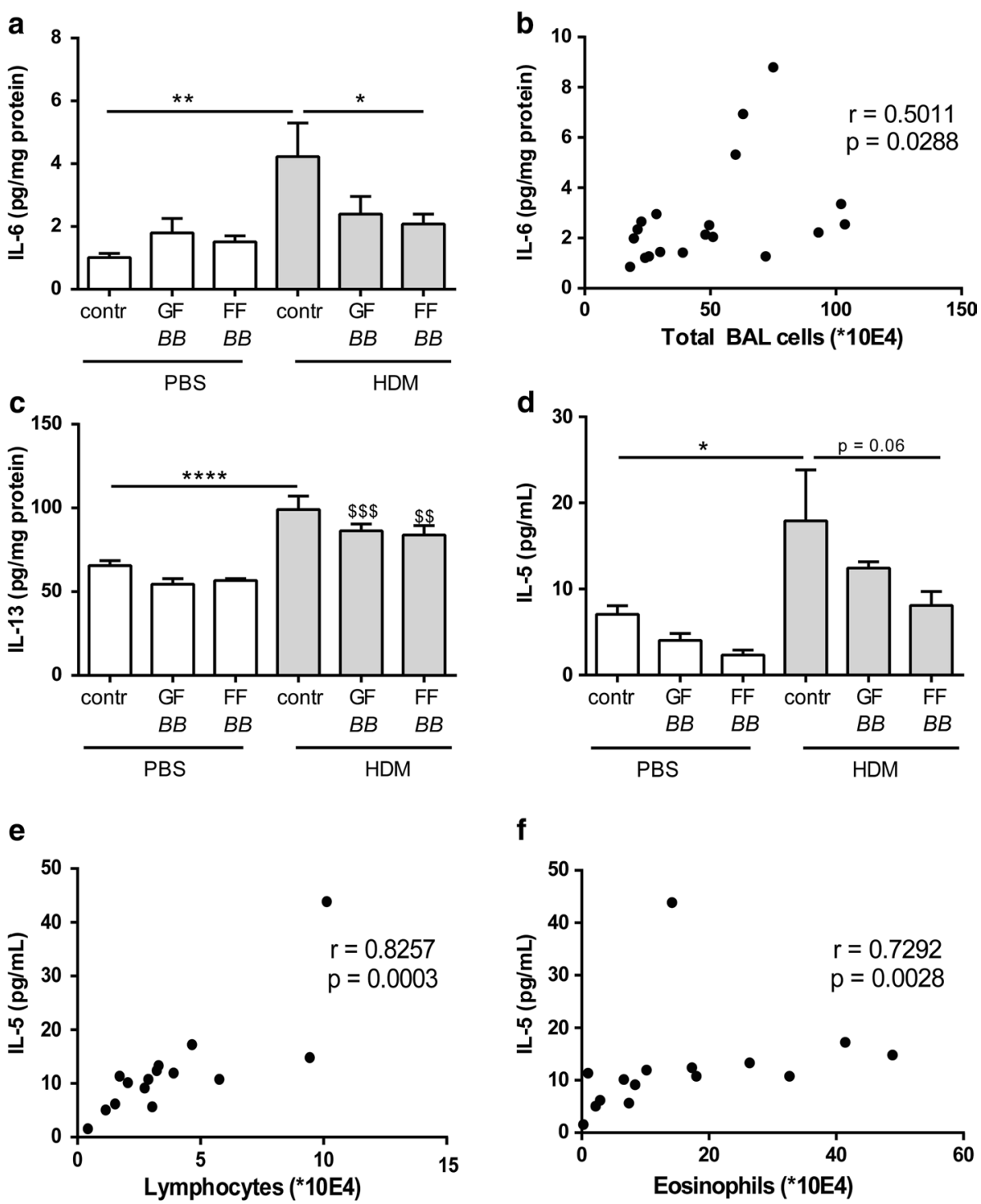

f

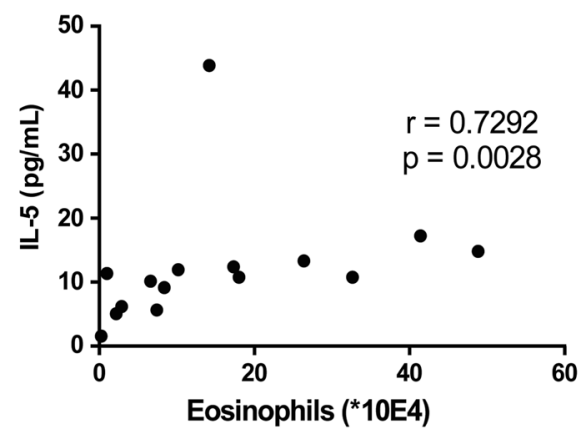

HDM stimulation, which activates DC [26] and is a chemoattractant for Th2 cells [27, 28]. IL-33 has also been shown to be increased in bronchial biopsies of asthmatic patients compared to non-asthmatic patients [29]. In the present study, a significant increase in IL-33 concentrations was observed in lung homogenates obtained from HDMallergic mice and no evidence was found that the dietary interventions modulated this increase.

Another relevant chemokine that is produced by the epithelium is CCL20 which is responsible for the attraction of immature DC to the lung [26, 30]. From human studies, it is known that CCL20 levels are increased in asthmatic patients compared to healthy controls, which is even more pronounced after allergen challenge [31]. In the current study, a significant increase in CCL20 in lung homogenates of the HDM-HDM mice compared to the HDM-PBS mice fed the control diet was found, which remained unaffected by the synbiotic diets.
In patients suffering from atopic asthma, it was shown that after a challenge with HDM, the concentrations of CCL17 were also increased in the BALF [32]. CCR4 and its ligand CCL17 are up-regulated in the airways of asthmatic patients after challenge and contribute to the Th2 cell recruitment in asthma $[32,33]$. However, CCR4 is not only a ligand for CCL17, but it also binds CCL22 with an even higher affinity [34]. CCR4 is expressed by regulatory $\mathrm{T}$ cells, mast cells and Th2 cells and is known to play a pivotal role in allergic diseases. In bronchial biopsies of asthmatic patients, most of the T lymphocytes were CCR4 positive [35]. In the current study, a significant increase in CCL17 and CCL22 was found in lung tissue of the HDMHDM mice compared to the HDM-PBS mice fed the control diet. However, the synbiotic diets did not affect these pulmonary concentrations of CCL17 and CCL22. Overall, these results indicate that although the FFBB diet effectively reduced lung eosinophilia and lymphocyte influx, the 

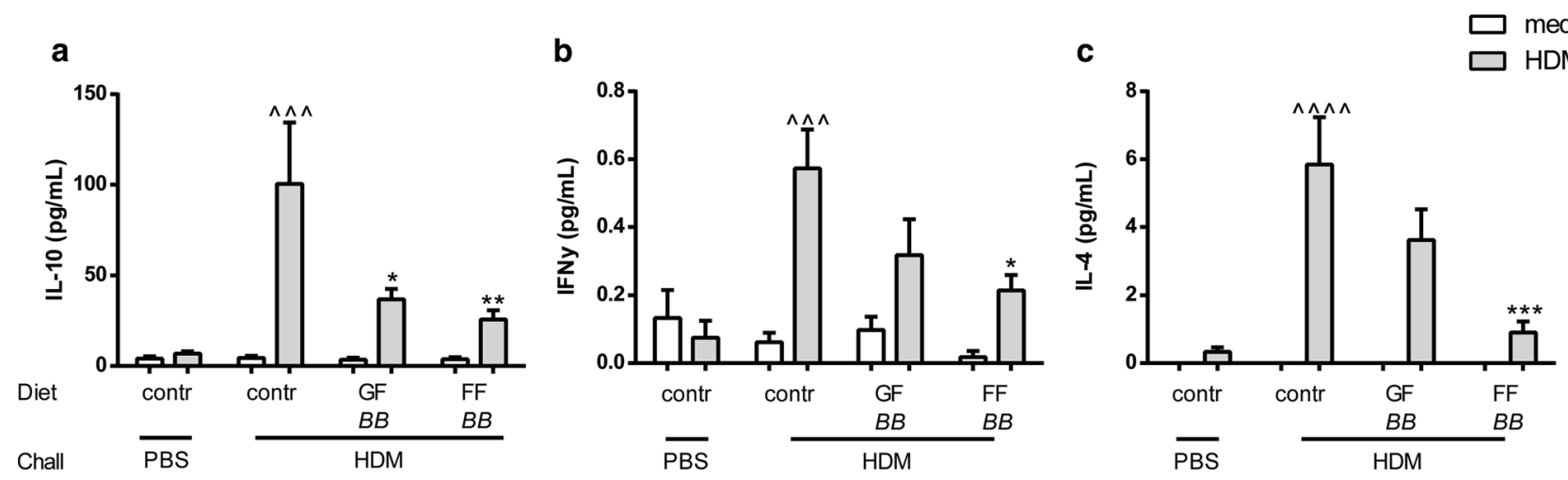

Fig. 5 Synbiotic diets suppress antigen-specific $\mathrm{T}$ cell activation in HDM-allergic mice. Lung cell suspensions were ex vivo restimulated with HDM for 4 days $\left(37{ }^{\circ} \mathrm{C}, 5 \% \mathrm{CO}_{2}\right)$. IL-10 (a), IFN $\gamma(\mathbf{b})$ and IL-4 (c) concentrations were measured in the supernatant. HDMPBS: HDM-sensitized and PBS-challenged mice (white bars), HDMHDM: HDM-sensitized and HDM-challenged mice (gray bars), Contr: control diet, GFBB: mixture of GOS and long-chain fructooligosaccharide with Bifidobacterium breve M-16V diet, FFBB:

diet did not suppress release of Th2-polarizing mediators by epithelial cells and/or DC.

IL-6 is produced not only by inflammatory cells such as DC and macrophages, but also by lung epithelial cells after stimulation with an allergen. The murine model for HDMinduced allergic asthma showed elevated pulmonary IL-6 concentrations, which is in line with human studies that showed elevated IL-6 levels in BALF, sputum and serum from asthmatic patients $[36,37]$. Dietary intervention with FFBB significantly decreased the concentration of IL-6 in lung homogenates, which correlated with the observed decrease in the influx of inflammatory cells. Hence, the decrease in IL-6 by the FFBB diet may relate to the protective effect of this diet on lung inflammatory responses as seen in this animal model.

Besides the pro-inflammatory IL-6, IL-13 and IL-5 are also investigated. These allergy-driven cytokines were secreted by antigen-specific Th2 cells and mast cells as well as by ILCs. IL-13 is also capable of triggering eosinophils and macrophages [38], and IL-5 is a Th2 cytokine which is essential for the maturation and activation of eosinophils [39]. The concentration of IL-13 in lung tissue and IL-5 in the BALF was significantly increased in HDMallergic mice compared to control mice. This effect corresponds to human studies which showed that IL-13 levels were increased in sputum of asthmatic patients [38, 40, 41]. Furthermore, IL-5 mRNA levels are increased in bronchial biopsies of asthmatic patients compared to healthy controls [42]. Since IL-5 is essential for eosinophil maturation and eosinophils are important in asthma, animal and human studies showed that eosinophilia in blood and BALF can be reduced with monoclonal antibodies against IL-5 [39, mixture of short-chain fructo-oligosaccharide and long-chain fructooligosaccharide with Bifidobacterium breve M-16V diet. Results are shown as mean \pm SEM. Statistical significance of differences by one-way ANOVA and post hoc Bonferroni's multiple comparisons test. $* P<0.05$, $* * P<0.01$, $* * * P<0.001$ compared to HDM-HDM; ${ }^{\wedge \wedge} P<0.001,{ }^{\wedge \wedge \wedge} P<0.0001$ compared to HDM-PBS, $n=6-7$ mice/ group

43]. Although $\mathrm{FFBB}$ did not reduce the concentration of IL-13 in lung homogenates, it showed a strong tendency to decrease the concentration of IL-5. Moreover, IL-5 in BALF was positively correlated with eosinophil and lymphocyte numbers. The reduction in inflammatory cell influx by FFBB may be a consequence of mechanisms involving the suppression of challenge-induced IL- 6 and IL- 5 secretion by lung inflammatory cells.

The FFBB diet suppressed lymphocyte influx, and indeed ex vivo antigen-specific restimulation of lung cell suspensions showed reduced Th1 (IFN $\gamma$ )- and Th2 (IL4)-type cytokine release by lung cells of HDM-challenged mice fed the FFBB diet. IFN $\gamma$ is a Th1 cytokine which is found to be increased in BALF and peripheral blood of asthmatic patients. In mouse models, IFN $\gamma$ is increased at mRNA levels in the airway epithelium [44]. IL-4 is one of the key players in the development of allergic asthma. A high IL-4 milieu is necessary for the differentiation of Th2 cells that in turn can also produce IL-4 upon antigen binding [26]. In patients with asthma, increased IL-4 protein levels were found in BALF and serum [45]. When patients with mild asthma were nebulized with IL-4, an elevation of eosinophil number in the sputum was observed. In addition, it was shown that after nebulizing with anti-IL-4 antibodies, eosinophilic inflammation was reduced in mice [46]. Since both allergen-induced IFN- $\gamma$ and IL-4 were decreased after restimulation ex vivo with HDM in the FFBB group, these results support the hypothesis that the influx of inflammatory cells into the lung tissue upon the previous in vivo allergen challenge was reduced by the diet.

The FFBB diet may be capable of reducing the allergenspecific effector response, which can include regulatory 


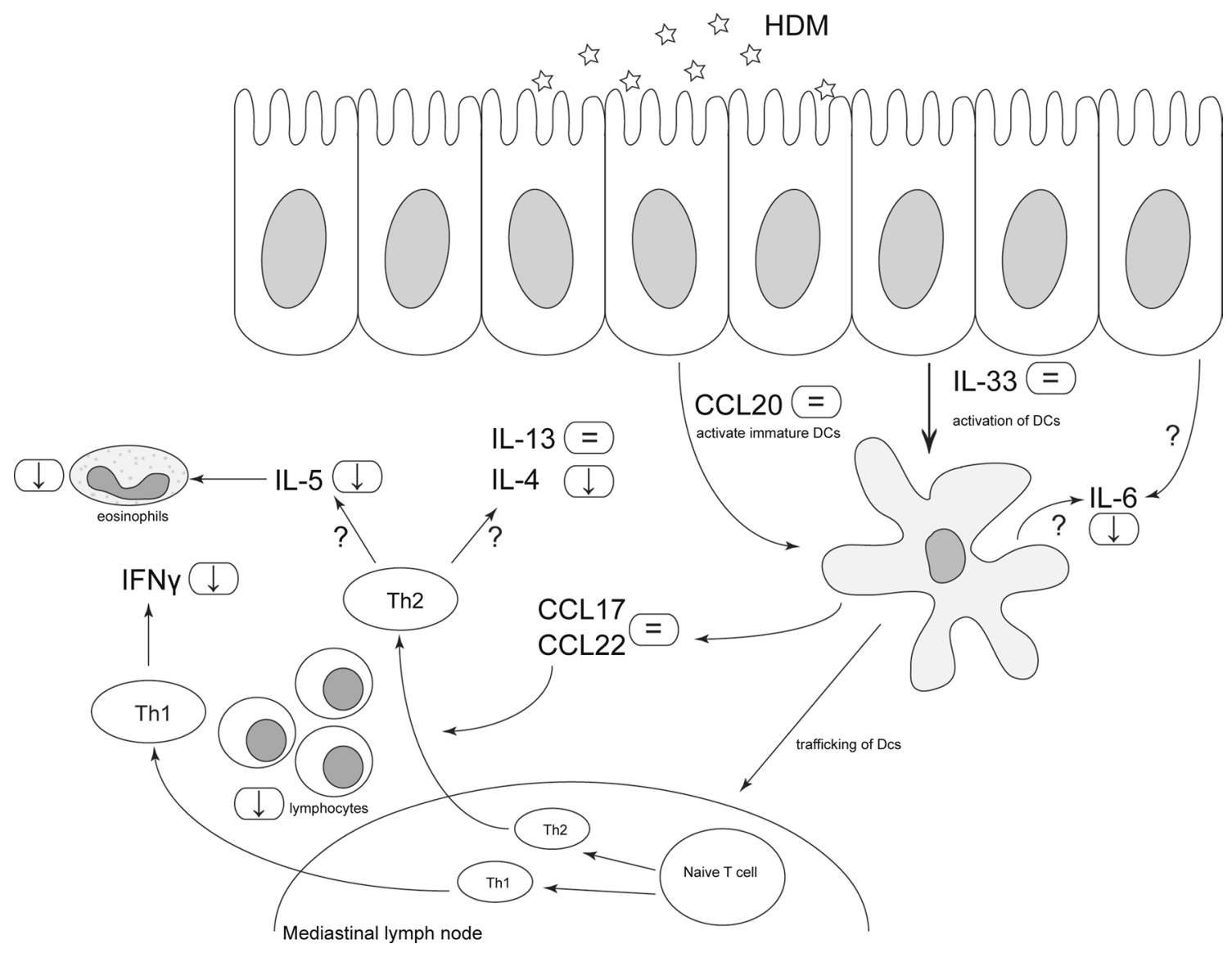

Fig. 6 Overview of the effects of the synbiotic diet FFBB. After the initial exposure to HDM, CCL20 is secreted by the airway epithelium, which will activate immature DCs. IL-33, also secreted by the epithelium, activates the DCs. Both CCL20 and IL-33 concentrations in lung homogenate supernatants were not affected by the FFBB diet. Activated DCs secrete CCL17 and CCL22, which are chemo-attractants for Th2 cells, and will traffic to the mediastinal lymph nodes to differentiate naïve $\mathrm{T}$ cells into Th2 cells. The FFBB diet did not affect CCL17 and CCL22 release by these DC. In contrast, IL-6, which

cytokines such as IL-10. Upon ex vivo HDM stimulation, IL-10 was also decreased in FFBB-fed group. Besides, FF $B B$ also GFB $B$ reduced IL-10, whereas IFN- $\gamma$ and IL-4 were not significantly lowered by GFBB. This may imply that GFBB acts via a different mechanism of action in particular targeting HDM-induced increase in IL-10 or that $\mathrm{FF} B B$ is more effective in dampening the inflammatory cascade.

Hence, the current study shows a protective effect of dietary FFBB in HDM-induced airway inflammation in mice when provided before and during allergic sensitization and challenge. FFBB did not affect IL-33 and CCL20 induction, which are known to initiate the development and activation of CCL17- and CCL22-producing Th2-polarizing DC contributing to allergic sensitization. However, FFBB may have reduced the influx of eosinophils and allergenspecific $\mathrm{T}$ cells by suppressing local secretion of IL- 6 and can be secreted by lung epithelial cells or inflammatory cells such as DC, was suppressed which correlated with the reduced inflammatory cell influx. Mainly lymphocyte and eosinophil influx was lowered by the FFBB diet. Among other cells, Th2 cells secrete IL-13, IL-4 and IL- 5 and Th1 cells secrete IFN- $\gamma$. Although IL-13 remained high, the FF $B B$ diet reduced IL- 4 and IFN $\gamma$. Furthermore, FF $B B$ tended to suppress IL-5 levels which correlated with the reduced number of lymphocytes and eosinophils upon dietary intervention

IL-5 by inflammatory cells via currently unknown mechanisms (Fig. 6).

Despite the strong effect of the FFBB diet on airway inflammation, it remains to be assessed whether FFBB may improve the clinical outcome (asthma) since the effects of FFBB on the AHR remained unclear in the current study. Future studies are needed to further optimize the effectivity of FFBB in prevention and/or treatment of HDM-induced asthma. This may include and earlier introduction prior to sensitization and dose optimization. Furthermore, combining FF with other, bifidobacteria or lactobacillus strains may be considered. Indeed in other mice studies, positive effects of L. rhamnosus have been observed on AHR and lung inflammation [13, 47]. A combination of FF with this or other bacteria might have beneficial effects on allergic asthmatic features. The mode of action of FFBB in suppressing lymphocyte and eosinophil influx hereby dampening the allergic 
asthma pathway is currently unclear. We hypothesize that compared to GFBB, FFBB changes the microbiota in a different manner and via these changes differentially affects the mucosal and systemic immune response. One of the ways by which this may occur is via altering the levels or patterns of short-chain fatty acids (SCFA) produced upon bacterial fermentation of the oligosaccharides in the intestine. These SCFA become available in the blood stream and have anti-inflammatory properties by acting via the GPR41 and GPR43 receptors and are known to suppress airway inflammation [48]. Indeed, SCFA have been shown to dampen asthmatic responses in HDM-allergic mice fed a high-fiber diet by lowering the number of eosinophils and lymphocytes and the concentration of Th2-related cytokines [49]. However, whether this also underlies the mechanism of FFBB needs to be further elucidated. The promising results of our study suggest the potential clinical application of this intervention, and in future studies the use of $\mathrm{FFBB}$ as an adjunct therapy for budesonide treatment, a corticosteroid used to treat asthma in humans, may be considered as well.

In conclusion, dietary intervention with a synbiotic supplementation can suppress pulmonary inflammation in HDM asthmatic mice, although the FFBB showed a more beneficial effect on the management of HDM-induced allergic asthma compared to GFBB. The mode of action of FFBB in suppressing airway eosinophilia and lymphocyte numbers hereby dampening the allergic asthma pathway needs to be further elucidated, but the promising results of our study suggest the potential clinical application of this intervention.

Acknowledgments This project is jointly financed by the European Union, European Regional Development Fund and The Ministry of Economic Affairs, Agriculture and Innovation, Peaks in the Delta, the Municipality of Groningen, the Provinces of Groningen, Fryslân and Drenthe, the Dutch Carbohydrate Competence Center (CCC WP25; www.cccresearch.nl), Nutricia Research and FrieslandCampina.

Conflict of interest LEMW, TLM, ADK and GF have no interest to declare. KATV and SB were granted by the Carbohydrate Competence Center (CCC) program as indicated in acknowledgements; JG and PVJ are employees of Nutricia Research, which is the industrial partner in the Dutch Carbohydrate Competence Center.

Open Access This article is distributed under the terms of the Creative Commons Attribution 4.0 International License (http://creativecommons.org/licenses/by/4.0/), which permits unrestricted use, distribution, and reproduction in any medium, provided you give appropriate credit to the original author(s) and the source, provide a link to the Creative Commons license, and indicate if changes were made.

\section{References}

1. WHO (2013) http://www.who.int/mediacentre/factsheets/fs307/ en/index.html. Accessed Apr 2014

2. Holgate ST (2012) Innate and adaptive immune responses in asthma. Nat Med 18:673-683
3. Dumez ME, Herman J, Campizi V, Galleni M, Jacquet A, Chevigne A (2014) Orchestration of an uncommon maturation cascade of the house dust mite protease allergen quartet. Front Immunol 5:138

4. Jacquet A (2013) Innate immune responses in house dust mite allergy. ISRN Allergy 2013:735031

5. Salazar F, Ghaemmaghami AM (2013) Allergen recognition by innate immune cells: critical role of dendritic and epithelial cells. Front Immunol 4:356

6. Li BW, Hendriks RW (2013) Group 2 innate lymphoid cells in lung inflammation. Immunology 140:281-287

7. Willart M, Hammad H (2011) Lung dendritic cell-epithelial cell crosstalk in Th2 responses to allergens. Curr Opin Immunol 23:772-777

8. Kudo M, Ishigatsubo Y, Aoki I (2013) Pathology of asthma. Front Microbiol 4:263

9. Gourbeyre P, Denery S, Bodinier M (2011) Probiotics, prebiotics, and synbiotics: impact on the gut immune system and allergic reactions. J Leukoc Biol 89:685-695

10. Noverr MC, Huffnagle GB (2004) Does the microbiota regulate immune responses outside the gut? Trends Microbiol 12:562-568

11. Vieira WA, Pretorius E (2010) The impact of asthma on the gastrointestinal tract (GIT). J Asthma Allergy 3:123-130

12. Kranich J, Maslowski KM, Mackay CR (2011) Commensal flora and the regulation of inflammatory and autoimmune responses. Semin Immunol 23:139-145

13. Sagar S, Morgan ME, Chen S, Vos AP, Garssen J, van Bergenhenegouwen J, Boon L, Georgiou NA, Kraneveld AD, Folkerts $\mathrm{G}$ (2014) Bifidobacterium breve and Lactobacillus rhamnosus treatment is as effective as budesonide at reducing inflammation in a murine model for chronic asthma. Respir Res 15:46

14. Gibson GR, Roberfroid MB (1995) Dietary modulation of the human colonic microbiota: introducing the concept of prebiotics. J Nutr 125:1401-1412

15. Slavin J (2013) Fiber and prebiotics: mechanisms and health benefits. Nutrients 5:1417-1435

16. Verheijden KA, Willemsen LE, Braber S, Leusink-Muis T, Delsing DJ, Garssen J, Kraneveld AD, Folkerts G (2015) Dietary galacto-oligosaccharides prevent airway eosinophilia and hyperresponsiveness in a murine house dust mite-induced asthma model. Respir Res 16:17

17. Schouten B, van Esch BC, Hofman GA, van Doorn SA, Knol J, Nauta AJ, Garssen J, Willemsen LE, Knippels LM (2009) Cow milk allergy symptoms are reduced in mice fed dietary synbiotics during oral sensitization with whey. J Nutr 139:1398-1403

18. Sagar S, Vos AP, Morgan ME, Garssen J, Georgiou NA, Boon L, Kraneveld AD, Folkerts G (2014) The combination of Bifidobacterium breve with non-digestible oligosaccharides suppresses airway inflammation in a murine model for chronic asthma. Biochim Biophys Acta 1842:573-583

19. van de Pol MA, Lutter R, Smids BS, Weersink EJ, van der Zee JS (2011) Synbiotics reduce allergen-induced T-helper 2 response and improve peak expiratory flow in allergic asthmatics. Allergy 66:39-47

20. Kool M, Willart MA, van Nimwegen M, Bergen I, Pouliot P, Virchow JC, Rogers N, Osorio F, Reis e Sousa C, Hammad H, Lambrecht BN (2011) An unexpected role for uric acid as an inducer of $\mathrm{T}$ helper 2 cell immunity to inhaled antigens and inflammatory mediator of allergic asthma. Immunity 34:527-540

21. Sagar S, Verheijden KA, Georgiou NA, Garssen J, Kraneveld AD, Vos AP, Folkerts G (2013) Differential regulation of inflammation and immunity in mild and severe experimental asthma. Mediat Inflamm 2013:808470

22. Braber S, Koelink PJ, Henricks PA, Jackson PL, Nijkamp FP, Garssen J, Kraneveld AD, Blalock JE, Folkerts G (2011) 
Cigarette smoke-induced lung emphysema in mice is associated with prolyl endopeptidase, an enzyme involved in collagen breakdown. Am J Physiol Lung Cell Mol Physiol 300:L255-L265

23. Koelink PJ, Overbeek SA, Braber S, Morgan ME, Henricks PA, Roda MA, Verspaget HW, Wolfkamp SC, Te Velde AA, Jones CW, Jackson PL, Blalock JE, Sparidans RW, Kruijtzer JA, Garssen J, Folkerts G, Kraneveld AD (2013) Collagen degradation and neutrophilic infiltration: a vicious circle in inflammatory bowel disease. Gut 63:578-587

24. Gregory LG, Lloyd CM (2011) Orchestrating house dust miteassociated allergy in the lung. Trends Immunol 32:402-411

25. Nials AT, Uddin S (2008) Mouse models of allergic asthma: acute and chronic allergen challenge. Dis Model Mech $1: 213-220$

26. Vroman H, van den Blink B, Kool M (2015) Mode of dendritic cell activation: the decisive hand in Th2/Th17 cell differentiation. Implications in asthma severity? Immunobiology 220:254-261

27. Oboki K, Nakae S, Matsumoto K, Saito H (2011) IL-33 and airway inflammation. Allergy Asthma Immunol Res 3:81-88

28. Borish L, Steinke JW (2011) Interleukin-33 in asthma: how big of a role does it play? Curr Allergy Asthma Rep 11:7-11

29. Makrinioti H, Toussaint M, Jackson DJ, Walton RP, Johnston SL (2014) Role of interleukin 33 in respiratory allergy and asthma. Lancet Respir Med 2:226-237

30. Zijlstra GJ, Fattahi F, Rozeveld D, Jonker MR, Kliphuis NM, van den Berge M, Hylkema MN, ten Hacken NH, van Oosterhout AJ, Heijink IH (2014) Glucocorticoids induce the production of the chemoattractant CCL20 in airway epithelium. Eur Respir J 44:361-370

31. Pichavant M, Charbonnier AS, Taront S, Brichet A, Wallaert B, Pestel J, Tonnel AB, Gosset P (2005) Asthmatic bronchial epithelium activated by the proteolytic allergen Der $\mathrm{p} 1$ increases selective dendritic cell recruitment. J Allergy Clin Immunol 115:771-778

32. Pilette C, Francis JN, Till SJ, Durham SR (2004) CCR4 ligands are up-regulated in the airways of atopic asthmatics after segmental allergen challenge. Eur Respir J 23:876-884

33. Vijayanand P, Durkin K, Hartmann G, Morjaria J, Seumois G, Staples KJ, Hall D, Bessant C, Bartholomew M, Howarth PH, Friedmann PS, Djukanovic R (2010) Chemokine receptor 4 plays a key role in $\mathrm{T}$ cell recruitment into the airways of asthmatic patients. J Immunol 184:4568-4574

34. Santulli-Marotto S, Boakye K, Lacy E, Wu SJ, Luongo J, Kavalkovich K, Coelho A, Hogaboam CM, Ryan M (2013) Engagement of two distinct binding domains on CCL17 is required for signaling through CCR4 and establishment of localized inflammatory conditions in the lung. PLoS One 8:e81465

35. Viney JM, Andrew DP, Phillips RM, Meiser A, Patel P, Lennartz-Walker M, Cousins DJ, Barton NP, Hall DA, Pease JE (2014) Distinct conformations of the chemokine receptor
CCR4 with implications for its targeting in allergy. J Immunol 192:3419-3427

36. Tillie-Leblond I, Pugin J, Marquette CH, Lamblin C, Saulnier F, Brichet A, Wallaert B, Tonnel AB, Gosset P (1999) Balance between proinflammatory cytokines and their inhibitors in bronchial lavage from patients with status asthmaticus. Am J Respir Crit Care Med 159:487-494

37. Yokoyama A, Kohno N, Fujino S, Hamada H, Inoue Y, Fujioka S, Ishida S, Hiwada K (1995) Circulating interleukin-6 levels in patients with bronchial asthma. Am J Respir Crit Care Med 151:1354-1358

38. Ingram JL, Kraft M (2012) IL-13 in asthma and allergic disease: asthma phenotypes and targeted therapies. J Allergy Clin Immunol 130:829-842 quiz 843-844

39. Greenfeder S, Umland SP, Cuss FM, Chapman RW, Egan RW (2001) Th2 cytokines and asthma. The role of interleukin-5 in allergic eosinophilic disease. Respir Res 2:71-79

40. Wills-Karp M (2004) Interleukin-13 in asthma pathogenesis. Immunol Rev 202:175-190

41. Saha SK, Berry MA, Parker D, Siddiqui S, Morgan A, May R, Monk P, Bradding P, Wardlaw AJ, Pavord ID, Brightling CE (2008) Increased sputum and bronchial biopsy IL-13 expression in severe asthma. J Allergy Clin Immunol 121:685-691

42. Kouro T, Takatsu K (2009) IL-5- and eosinophil-mediated inflammation: from discovery to therapy. Int Immunol 21:1303-1309

43. Schuijs MJ, Willart MA, Hammad H, Lambrecht BN (2013) Cytokine targets in airway inflammation. Curr Opin Pharmacol 13:351-361

44. Kumar RK, Yang M, Herbert C, Foster PS (2012) Interferongamma, pulmonary macrophages and airway responsiveness in asthma. Inflamm Allergy Drug Targets 11:292-297

45. Maes T, Joos GF, Brusselle GG (2012) Targeting interleukin-4 in asthma: lost in translation? Am J Respir Cell Mol Biol 47:261-270

46. Steinke JW, Borish L (2001) Th2 cytokines and asthma. Interleukin-4: its role in the pathogenesis of asthma, and targeting it for asthma treatment with interleukin- 4 receptor antagonists. Respir Res 2:66-70

47. Wu CT, Chen PJ, Lee YT, Ko JL, Lue KH (2014) Effects of immunomodulatory supplementation with Lactobacillus rhamnosus on airway inflammation in a mouse asthma model. $\mathrm{J}$ Microbiol Immunol Infect. doi:10.1016/j.jmii.2014.08.001

48. Vinolo MA, Rodrigues HG, Nachbar RT, Curi R (2011) Regulation of inflammation by short chain fatty acids. Nutrients 3:858-876

49. Trompette A, Gollwitzer ES, Yadava K, Sichelstiel AK, Sprenger N, Ngom-Bru C, Blanchard C, Junt T, Nicod LP, Harris NL (2014) Gut microbiota metabolism of dietary fiber influences allergic airway disease and hematopoiesis. Nat Med 20:159-166 